\title{
Study of band structure, transport and magnetic properties of $\mathrm{BiFeO}_{3}-\mathrm{TbMnO}_{3}$ composite
}

\author{
Prince K. Gupta ${ }^{1}$ Surajit Ghosh ${ }^{1} \cdot$ Arkadeb Pal $^{1} \cdot$ Somnath Roy $^{2} \cdot$ Amish G. Joshi $^{3} \cdot$ A. K. Ghosh ${ }^{2} \cdot$ Sandip Chatterjee $^{1}$
}

Received: 13 August 2019 / Accepted: 5 November 2019 / Published online: 12 November 2019

(c) Springer Nature Switzerland AG 2019

\begin{abstract}
Charge transfer across the interface of two materials in a composite can create reconstruction of bands near the interface which in turn brings multiple changes in physical properties of the materials. Thus, investigation of band structure experimentally is of immense importance in studying composite materials to understand their physical properties. Here, we have studied magnetoelectric multiferroic composite of two types of multiferroic (types I and II) consisting of $\mathrm{BiFeO}_{3}$ and $\mathrm{TbMnO}_{3}$ for enhanced magnetic and transport properties. The band structure was investigated with the help of UV-visible absorption spectrum, the valence band X-ray photoemission spectra (XPS), and ultraviolet photoemission spectra. The band structure thus obtained can successfully explain the magnetic and transport properties of the composite. The insulating behavior of the system is understood from the reconstruction of the energy bands at the interface and subsequent decrease in the band gap which happens due to lattice mismatch of the two materials. The large coercivity and the increase in the magnetization value are understood to be due to superexchange interaction between different $\mathrm{Mn}$ ions $\left(\mathrm{Mn}^{2+}, \mathrm{Mn}^{3+}\right.$, and $\left.\mathrm{Mn}^{4+}\right)$. From the composition study of EDXA and core-level XPS, oxygen vacancy was found which in turn creates the mixed valence state of $\mathrm{Mn}$ to maintain the charge neutrality.
\end{abstract}

Keywords $\mathrm{BiFeO}_{3} \cdot \mathrm{TbMnO}_{3} \cdot$ Band structure $\cdot$ Magnetoelectric multiferroic

\section{Introduction}

Multiferroic materials have been attracting researchers recently for their interesting fundamentals as well as for the possibility of application of these materials in different spintronic devices [1-4]. In magnetoelectric multiferroic materials, ferroic (or anti-ferroic) magnetic and electric ordering coexist in a single phase giving rise to the possibility of controlling the magnetization (intrinsic polarization) with the application of electric field (magnetic field) [1]. Due to these coupling between the two properties, magnetoelectric multiferroic materials have become one of the most important materials of today [1]. The reason for the limited number of multiferroic material is the mutual exclusive origin of the two ordering (empty $d$ shell for ferroelectricity and partially filled $\mathrm{d}$ shell for magnetic ordering) [1-4].

$\mathrm{BiFeO}_{3}$ is one of the most interesting and well-studied multiferroic as it is the only multiferroic material to show both the ordering (magnetic and ferroelectric) above the room temperature (ferroelectric Curie temperature $T_{\mathrm{N}} \sim$ $1103 \mathrm{~K}$ and Neel temperature $T_{\mathrm{N}} \sim 643 \mathrm{~K}$ ) [5-8]. It exhibits G-type canted antiferromagnetic ordering with a cycloid frequency of $\sim 62 \mathrm{~nm}$ [8]. $\mathrm{BiFeO}_{3}$ shows large spontaneous polarization of order $10-100 \mu \mathrm{c} / \mathrm{cm}^{2}$ because of polar displacement of cations and anions relative to each other pointing along one of the eight pseudo-cubic [111] axes [5-7]. The lone pair electron at the $6 \mathrm{~s}$ shell of $\mathrm{Bi}$ is

$\triangle$ Sandip Chatterjee, schatterji.app@iitbhu.ac.in | ${ }^{1}$ Department of Physics, Indian Institute of Technology (BHU) Varanasi, Varanasi 221005 , India. ${ }^{2}$ Department of Physics, Banaras Hindu University, Varanasi 221 005, India. ${ }^{3}$ CSIR-National Physical Laboratory, Dr. K. S. Krishnan Road, New Delhi 110 012, India. 
considered to be the main reason behind the observed ferroelectricity on $\mathrm{BiFeO}_{3}$ while the partially filled $3 d$ shell of $\mathrm{Fe}$ is responsible for the canted antiferromagnetic ordering [5-7]. $\mathrm{BiFeO}_{3}$ has been found to be useful in different modern-day technologies including microwave synthesis $[9,10]$. In spite of having these features, $\mathrm{BiFeO}_{3}$ is not considered suitable for many applications due to many reasons. Structural instability, difficulty in synthesizing single phase, high leakage current, and low resistivity of the material due to the presence of $\mathrm{Fe}^{3+}$ and oxygen vacancies are some of them [11-14]. To overcome the shortcomings, there have been numerous attempts involving doping of different transition metal at Fe site and rare earth metals at Bi sites, inducing chemical pressure or strain in the system [15-22]. An alternate option is to prepare composite of different multiferroic materials with a similar structure involving $\mathrm{BiFeO}_{3}$. There are reports on composite structures and superlattice structures of $\mathrm{BiFeO}_{3}-\mathrm{BaTiO}_{3}, \mathrm{BiFeO}_{3}-\mathrm{PbTiO}_{3}$, and $\mathrm{BiFeO}_{3}-\mathrm{BiMnO}_{3}$, showing improvement in multiferroic properties [23-25]. Yu et al. [26] have reported exchange bias and other enhanced magnetic properties in $\mathrm{BiFeO}_{3}-\mathrm{La}_{0.7} \mathrm{Sr}_{0.3} \mathrm{MnO}_{3}$ heterostructure due to charge transfer-assisted band reconstruction near the interface which in turn creates additional ferromagnetic and antiferromagnetic exchange interactions.

In this context, we have prepared a composite of $\mathrm{BiFeO}_{3}$ and $\mathrm{TbMnO}_{3}$, belonging to a different type of multiferroic. $\mathrm{TbMnO}_{3}$ is type II multiferroic material in which the ferroelectric ordering $\left(T_{C} \sim 28 \mathrm{~K}\right)$ arises as a result of magnetic ordering $\left(T_{\mathrm{N}} \sim 42 \mathrm{~K}\right)$, and the material is known to possess strong magnetoelectric coupling $[27,28]$. It crystallizes in orthorhombic perovskite $(\mathrm{Pbnm})$ close to that of $\mathrm{BiFeO}_{3}$ which has rhombohedral perovskite (R3C) structure $[27,29]$. The lattice mismatch between them is expected to trigger band reconstruction near the interface [26]. Band reconstruction can influence many physical properties of the composite including transport and magnetic properties. In one of our earlier reports, it was reported that the composite of $\mathrm{BiFeO}_{3}$ and $\mathrm{TbMnO}_{3}$ (in 7:3 ratio) $0.7 \mathrm{BFO}-0.3 \mathrm{TMO}$ shows interesting properties like spin-phonon coupling, magnetodielectric coupling, high dielectric constant, etc. [30]. It was also seen that charge accumulation and charge transfer at the interface of two materials of the composite in determining different properties of the composite. Thus, it would be significant to study the band structure of the composite and band reconstruction if any due to the charge transfer. It is also interesting to study the changes in transport and magnetic properties due to band reconstruction. In this report, we have studied the band structure through valence band $\mathrm{X}$-ray photoemission spectroscopy (XPS) and ultraviolet photoemission spectroscopy (UPS) and UV-visible absorption spectroscopy. The effect of band reconstruction on the transport and magnetic properties has also been studied.

\section{Materials and methods}

The compounds $\mathrm{BiFeO}_{3}, \mathrm{TbMnO}_{3}$, and the composite of $\mathrm{BiFeO}_{3}$ and $\mathrm{TbMnO}_{3}$ (in 7:3 ratio), i.e., 0.7BFO-0.3TMO, were prepared following a conventional solid-state reaction method by taking the precursors for all the constituent elements i.e., $\mathrm{Bi}_{2} \mathrm{O}_{3}, \mathrm{Fe}_{2} \mathrm{O}_{3}, \mathrm{Mn}_{2} \mathrm{O}_{3}$, and $\mathrm{Tb}_{4} \mathrm{O}_{7}$ (Alfa Aesar, USA) in proper stoichiometric ratio. At first, powders of $\mathrm{TbMnO}_{3}$ were prepared by solid-state reaction of $\mathrm{Tb}_{4} \mathrm{O}_{7}$ and $\mathrm{Mn}_{2} \mathrm{O}_{3}$ in proper stoichiometric ratio. The mixture was calcined $1200^{\circ} \mathrm{C}$ for $12 \mathrm{~h}$. The calcined powders were pelletized and sintered at 1300 and $1400^{\circ} \mathrm{C}$ for $24 \mathrm{~h}$. The powder was ground thoroughly in between. Then the $\mathrm{TbMnO}_{3}$ powder was mixed with $\mathrm{Bi}_{2} \mathrm{O}_{3}$ and $\mathrm{Fe}_{2} \mathrm{O}_{3}$ in stoichiometric ratio, and the mixture was ground with mortar pestle for $4 \mathrm{~h}$. The mixture was calcined at $900^{\circ} \mathrm{C}$ for $6 \mathrm{~h}$ after which it was pelletized and sintered for $10 \mathrm{~h}$ at $1000^{\circ} \mathrm{C}$ to get the final sample. The surface morphology and grain growth are studied from field emission scanning electron microscope (FESEM, Nova Nano SEM 450). The composition of the samples was also studied from energy-dispersive $X$-ray analysis (TEAM EDS SYSTEM with Octane Plus SDD Detector) integrated with the SEM. X-ray photoemission spectroscopy (XPS) experiments were performed using Omicron Multi-probe Surface Science System, GmBH, equipped with a dual-anode non-monochromatic Mg/ Al X-ray source (DAR400), a monochromatic source (XM 1000), and a hemispherical electron energy analyzer (EA 125). All the XPS measurements were performed inside the analysis chamber under base vacuum of $\sim 1.8 \times 10^{-10}$ Torr using monochoromatized $\mathrm{AlK}_{\mathrm{a}}$ with a power of $300 \mathrm{~W}$. The total energy resolution for monochromatic AlKa line with photon energy $1486.70 \mathrm{eV}$, estimated from the width of the Fermi edge, was about $0.25 \mathrm{eV}$. The pass energy for Survey Scan Spectra and core level spectra was kept at $50 \mathrm{eV}$ and $30 \mathrm{eV}$, respectively. Ultraviolet photoemission spectroscopy (UPS) and UV-visible absorption spectroscopy were employed to study valence band structure and electronic properties. To investigate the fine changes near Fermi level, Fermi edge ultraviolet photoemission spectra were collected using the non-monochromatic $\mathrm{He}$ I $(21.2 \mathrm{eV})$ line at an average base pressure of $2.8 \times$ $10^{-8}$ Torr. The energy resolution of the analyzer and the step size were set at $0.03 \mathrm{eV}$ and $0.05 \mathrm{eV}$, respectively. The magnetic measurements were carried out in Magnetic Property Measurement System (SQUID-MPMS, Quantum Design, USA). The temperature-dependent transport properties were measured in Close Cycle He cryostat 
(Advanced Research Systems, Inc.) using a Keithley 6517B Electrometer.

\section{Result and discussion}

In order to visualize surface morphology and microstructure of the composite images of sintered 0.7BFO-0.3TMO composite along with the pure BFO and TMO were recorded in the field emission scanning electron microscope (FESEM) (shown in Fig. 1a-c). It can be observed that pure BFO pellet exhibits rectangular and non-homogenously distributed grains with smooth surfaces showing crystal growth of BFO. The average grain size was calculated using ImageJ software with an average size of $2.16 \mu \mathrm{m}$. TMO microstructure consisted of non-homogenously distributed grains of an irregular shape with average grain size of $1.19 \mu \mathrm{m}$. The composite also shows similar morphology with irregular-shaped grains, but the grain size varies in broad range as compared to that of BFO and TMO grains. The average grain size also lies in between that of the two pure compounds $(1.59 \mu \mathrm{m})$ [31]. In order to identify all the elements present in 0.7BFO-0.3TMO composite, energy-dispersive X-ray analysis (EDXA) analysis was carried out, which is shown in Fig. 1d. The obtained EDXA result confirmed the homogenous mixing and phase formation of the composite. The EDXA result also indicated the presence of oxygen vacancy in the system. Manganite systems sintered at a higher temperature tend to have oxygen vacancies which in turn can influence the Mn ions present in the system to have multiple valence states in order to maintain the charge neutrality [32].

Fourier transform infrared (FTIR) spectroscopy has been one of the most used techniques these days to analyze different functional groups and corresponding vibrational bands. The FTIR spectrum of the composite $0.7 \mathrm{BFO}-0.3 \mathrm{TMO}$ was recorded in the wavenumber range of $400-2000 \mathrm{~cm}^{-1}$ which is presented in Fig. 2. The vibrational bands of metal-oxygen bonds generally lie below $1000 \mathrm{~cm}^{-1}$. In the case of pure $\mathrm{BiFeO}_{3}$, the bending and stretching vibrations of the $\mathrm{Fe}-\mathrm{O}$ in $\mathrm{FeO}_{6}$ octahedra and the vibrational band of $\mathrm{BiO}_{6}$ octahedra lie in the range from 400 to $560 \mathrm{~cm}^{-1}$, which has been clearly observed in the spectrum (peaks denoted as A and B) [33, 34]. The presence of $\mathrm{TbMnO}_{3}$ perovskite phase in the composite is confirmed by identifying the stretching vibration of
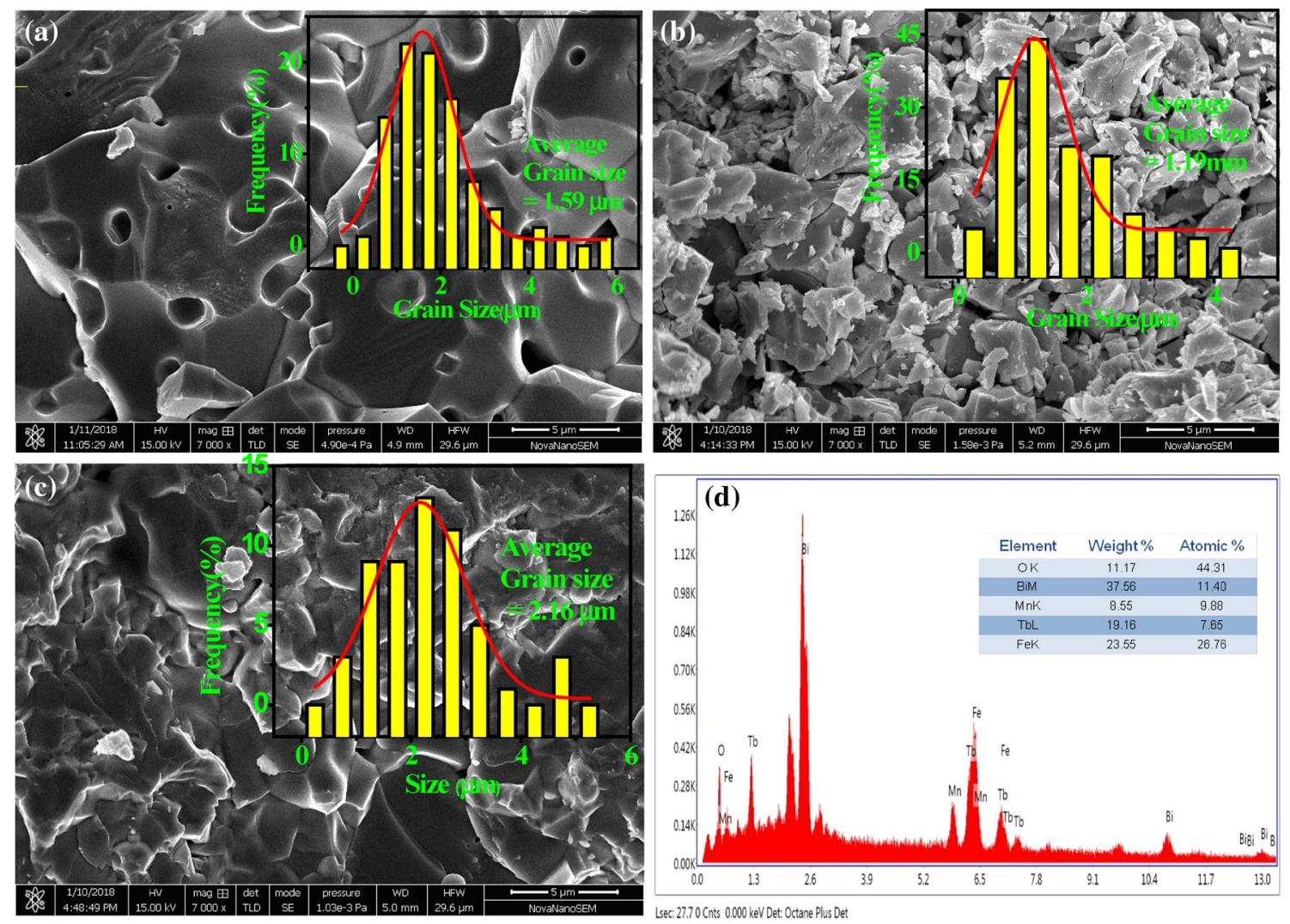

Fig. 1 a-c FESEM images from fractured surfaces of the singlephase BFO, TMO, and composite 0.7BFO-0.3TMO, respectively, and insets show the corresponding calculation of grains size bye image

j software. $\mathbf{d}$ shows EDXA graph of the composite 0.7BFO-0.3TMO with its chemical composition in the insets 


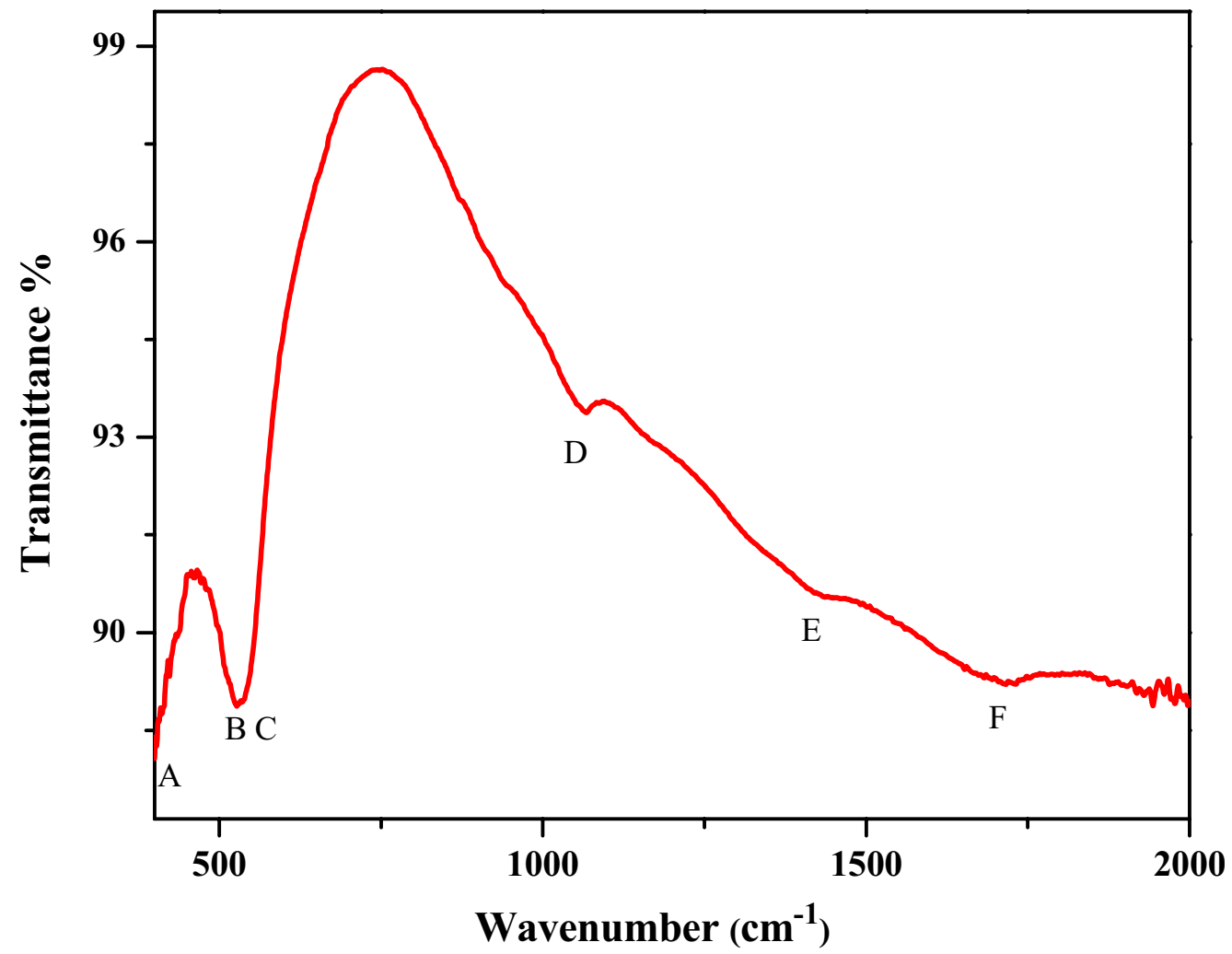

Fig. 2 FTIR spectrum of the composite 0.7BFO-0.3TMO recorded at $300 \mathrm{~K}$

$\mathrm{Mn}-\mathrm{O}-\mathrm{Mn}$ bond $\sim 573 \mathrm{~cm}^{-1}$ arising from $\mathrm{MnO}_{6}$ octahedra of the compound (denoted as $C$ in the spectrum). Apart from these bands few other bands can be identified in the spectrum such as $1720 \mathrm{~cm}^{-1}$ assigned to the bending vibrations of $\mathrm{H}_{2} \mathrm{O}$ which arises due to the presence of moistures in the powder $[33,35]$. The bands $\sim 1070 \mathrm{~cm}^{-1}$ and $1440 \mathrm{~cm}^{-1}$ are ascribed to the stretching vibrations of $C=O$ and $C-C$, respectively, which appears due to the absorption of carbon during the exposure of the sample to air [36].

X-ray photoemission spectroscopy (XPS) is a prominent technique followed by many researchers to study the chemical composition and valence state of constituent elements of material. We have studied the survey scan and detail scan of Fe2p, Mn2p, O1s, Bi4f, and Tb3d spectra to gain detail knowledge of their electronic structure and composition in the composite $\mathrm{BiFeO}_{3}-\mathrm{TbMnO}_{3}$. All the peak positions were matched with the National Institute of Standards and Technology (NIST) XPS database. From the survey, it was confirmed that all the constituent elements (i.e., $\mathrm{Bi}$. $\mathrm{Fe}, \mathrm{Tb}, \mathrm{Mn}$, and $\mathrm{O}$ ) were present in the composite (Fig. 3a). The absence of any foreign element in the compound was also confirmed from the spectrum. Although a $C 1 s$ peak can be seen from the survey scan spectrum, the presence of carbon does not influence any physical property of the composite. In many systems, such surface absorbed/adsorbed carbon has been found which does not have any effect on the physical properties. The detail spectra were deconvoluted using the XPSPEAK software. In Fig. 3b, the deconvoluted spectrum of the Fe2p core level is shown, which is split into two broad peaks due to spin-orbit coupling. The characteristic peaks of Fe2 $p_{3 / 2}$ and $\mathrm{Fe} 2 p_{1 / 2}$ are observed around the binding energies 710.6 and $724.7 \mathrm{eV}$, respectively, which correspond to $\mathrm{Fe}^{3+}$ state in BFO [37-39]. In addition to the characteristic peaks, there is a satellite peak, which is observed at the binding energy position $719 \mathrm{eV}$. The presence of the satellite peak $\sim 719 \mathrm{eV}$ confirms that trivalent oxidation state is the dominant state of iron in BFO which is expected for $\mathrm{BiFeO}_{3}[32,33]$. The XPS analysis indicates that only pure $\mathrm{BiFeO}_{3}$ phase is present without any type of impurities.

Figure $3 c$ shows the 01s XPS spectrum of the composite which is split into two parts revealing two kinds of chemical state of oxygen present in the composite. The peaks at binding energy position $\sim 529.3 \mathrm{eV}$ correspond to the oxygen situated at lattice denoted as $\mathrm{O}_{\mathrm{L}}$ and the peak positioned at $\sim 531.3 \mathrm{eV}$ corresponds to the surface chemisorbed oxygen denoted by $\mathrm{O}_{\mathrm{v}}$. The surface chemisorptions of the oxygen ions occur due to the presence of oxygen vacancy in the system. In $\mathrm{BiFeO}_{3}$-based compounds, it 

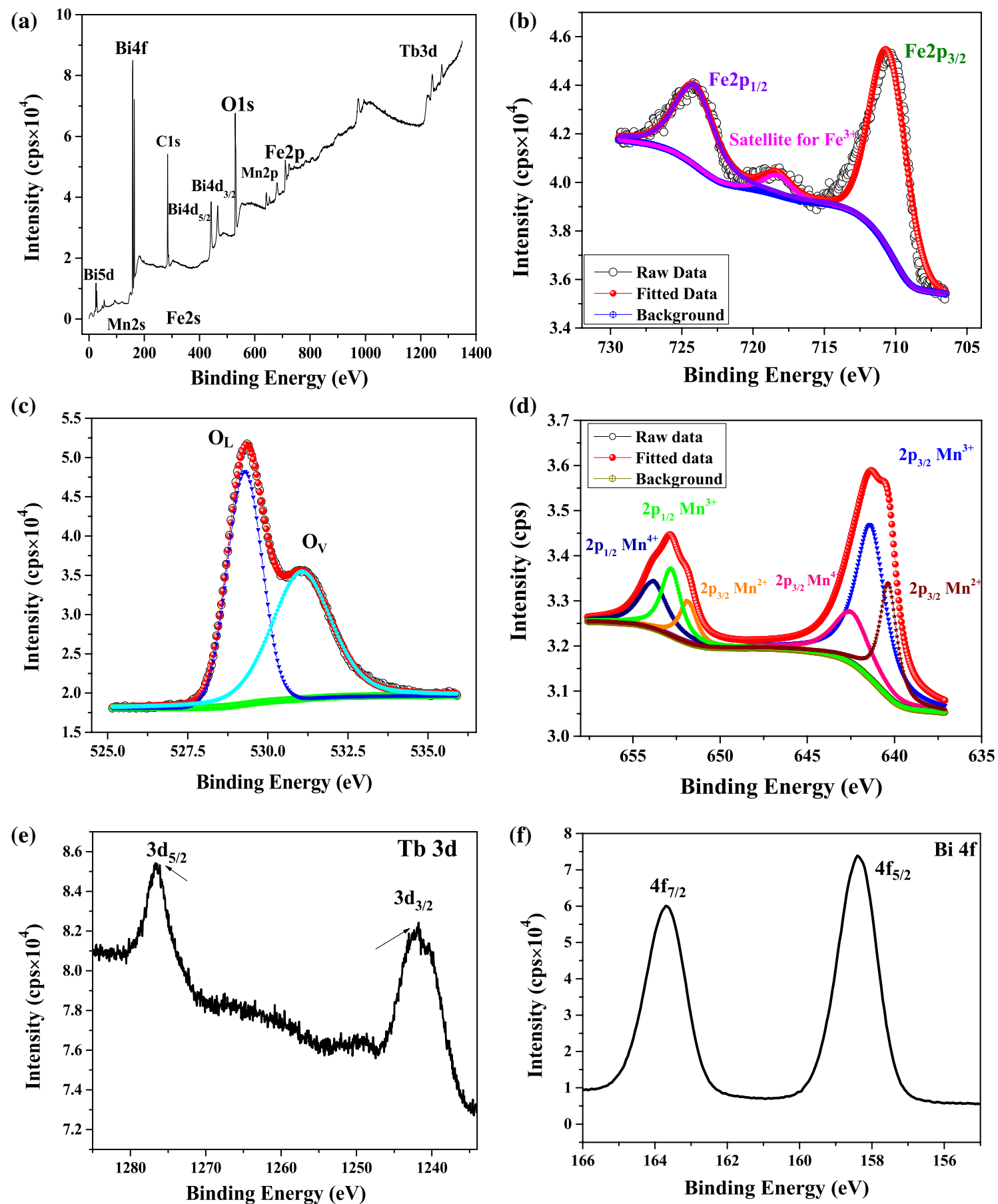

Fig. 3 a XPS survey scan of 0.7BFO-0.3TMO at $300 \mathrm{~K}$. Deconvoluted detail spectra of b Fe $2 p, \mathbf{c}$ O 1 s, d Mn 2p of composite $0.7 \mathrm{BFO}-0.3 \mathrm{TMO}$. Detail spectra of $\mathbf{e} \mathrm{Tb} 3 d$ and $\mathbf{f} \mathrm{Bi} 4 f$

is common to find the oxygen vacancies which are created at the surface due to lattice defects $[34,40]$. Moreover, our XPS results conform with EDX analysis which indicated the presence of oxygen vacancy in the system.

In Fig. $3 \mathrm{~d}$, the deconvoluted $\mathrm{Mn} 2 p$ spectrum is presented which is also found to be split into two characteristic spin-orbit peaks for $\mathrm{Mn} 2 p_{3 / 2}$ and $\mathrm{Mn} 2 p_{1 / 2} \sim 642.6$ and $654.0 \mathrm{eV}$, respectively. Interestingly, the peaks were asymmetric in nature indicating multiple valence states of $\mathrm{Mn}$ ions in the composite. The $\mathrm{Mn} 2 p_{3 / 2}$ and peak were deconvoluted into three characteristic peaks at binding energies $640.4,641.4$, and $642.6 \mathrm{eV}$, which correspond to $\mathrm{Mn}^{2+} \mathrm{Mn}^{3+}$ and $\mathrm{Mn}^{4+}$, respectively. The deconvoluted peak positions for the $\mathrm{Mn}^{2+}, \mathrm{Mn}^{3+}$, and $\mathrm{Mn}^{4+}$ species 
were found at $651.8,652.8$, and $654.0 \mathrm{eV}$, respectively. From the deconvoluted characteristic peaks, $\mathrm{Mn}^{3+}$ was found to be more intense, indicating dominant presence of $\mathrm{Mn}^{3+}$ which is obvious in case of $\mathrm{TbMnO}_{3}$. It is possible to estimate the fraction from the area under each curve representing a particular ion. The fractional composition is estimated using the relation:

$\%$ fraction $=\frac{\operatorname{Area}_{j} / s_{j}}{\sum \text { Area }_{j} / s_{j}}$,

where $S_{j}$ is the relative sensitivity factor. The relative fractions have been found to be $22.85 \%, 50.46 \%$, and $26.68 \%$ for the $\mathrm{Mn}^{2+}, \mathrm{Mn}^{3+}$, and $\mathrm{Mn}^{4+}$ respectively. The mixed phase of the $\mathrm{Mn}$ ions evolves in the system to maintain the charge neutrality which is affected by the oxygen vacancy observed from the $01 \mathrm{~s}$ detail spectrum. It has been observed that, in Mn-based systems, charge transfer effect between the Mn3d orbitals and $\mathrm{O} 2 p$ ligand orbitals can give rise to the mixed phase of $\mathrm{Mn}$ [ 41 and references therein]. The creation of $\mathrm{Mn}^{4+}$ and $\mathrm{Mn}^{2+}$ species can also be due to the valence instability of $\mathrm{Mn}^{3+}$ ions. The mix valence state of $\mathrm{Mn}$ ions is known to modify the bulk magnetic and electrical properties of different manganite systems [42].

In Fig. 3e, core level XPS spectra of Tb3d element is presented. The spectra show spin-orbit coupling peaks of $\mathrm{Tb}$ $3 d$ positioned at $\sim 1276.7 \mathrm{eV}$ and $\sim 1241.8 \mathrm{eV}$ for $3 d_{3 / 2}$ and $3 d_{5 / 2}$, respectively. The peak positions and the doublet separation clearly suggest that $\mathrm{Tb}$ is in $3+$ oxidation state [NIST]. The core-level spectra of Bi4f are also studied and are shown in Fig. 3f. The spin-orbit split peaks of Bi4f $f_{5 / 2}$ and $\mathrm{Bi} 4 \mathrm{f}_{7 / 2}$ are observed to be present at $\sim 163.7 \mathrm{eV}$ and $\sim 158.3 \mathrm{eV}$, which corroborate well with earlier reports of trivalent valence states of $\mathrm{Bi}[43,44]$.

The presence of different charge states of TM ions at the interface due to the charge transfer between $\mathrm{Mn}-\mathrm{Mn}$ ions may lead to the band reconstruction at the interface. As a result of the reconstruction, band gap of the composite is expected to decrease $[45,46]$. Thus to confirm the band gap reduction, we have measured the absorption spectrum in the UV and visible range. For studying the absorption characteristics of the composite, absorbance at different wavelengths ( $k$ ) (range of $200-800 \mathrm{~nm}$ ) was recorded and the absorption coefficients (a) were calculated at corresponding wavelengths. As can be seen from the spectrum, the absorption band edge lies beyond the range of measurement. We have followed the Tauc's method to estimate the band gap of the composite from the absorption spectra [26]. The photon energy (hv) and the band gap energy for a particular transition are related by the equation: $(\alpha h v)=K\left(h v-E_{\mathrm{g}}\right)^{n}$,

where $a$ is the absorption coefficient given by $a=2.303$ $(A b / t)$ (here, $A b$ is absorbance and $t$ is the thickness of the cuvette which is $1 \mathrm{~cm}$ ) and $K$ is the edge width parameter. The value of $\mathrm{n}$ depends on the type of transition, i.e., allowed direct, allowed indirect, forbidden direct, and forbidden indirect for which it can have values $1 / 2,2,3 / 2$, and 3 , respectively. $\mathrm{BiFeO}_{3}$ is known to have a band gap of $2.1 \mathrm{eV}$ to $2.7 \mathrm{eV}$ in different forms of the material such as bulk, nanomaterials, or single crystalline material [47-49]. Since $\mathrm{BiFeO}_{3}$ and $\mathrm{TbMnO}_{3}$ both are known to be direct band gap material, the band gap of the composite was determined from the linear fitting of the straight-line part of the $(a h v)^{2}$ versus photon energy $(h v)$ plot on the $h v$ axis [50]. From the Tauc's plot (Fig. 4), it is evident that the band gap of the material lies $\sim 01.45 \mathrm{eV}$, which is low compared to other reported values for $\mathrm{BiFeO}_{3}$ [47-49]. Therefore the reduction in the band gap of the composite system confirms the band reconstruction phenomenon due to the charge transfer between the TM ions in the composite.

The XPS valence band (VB) spectra of the 0.7BFO-0.3TMO system has been recorded at room temperature $(300 \mathrm{~K})$ to gain better insight into its detailed electronic structure (Fig. 5b). It is evident from the figure that near Fermi level $\left(E_{\mathrm{F}}=0 \mathrm{eV}\right)$, the spectral weight of the electronic states is extremely weak or merely absent which essentially suggests an insulating ground state of the system. Moreover, it is relevant here to mention that the UV-visible spectroscopy study yielded a high band gap of $\sim 1.45 \mathrm{eV}$ and the resistivity data exhibited an insulating

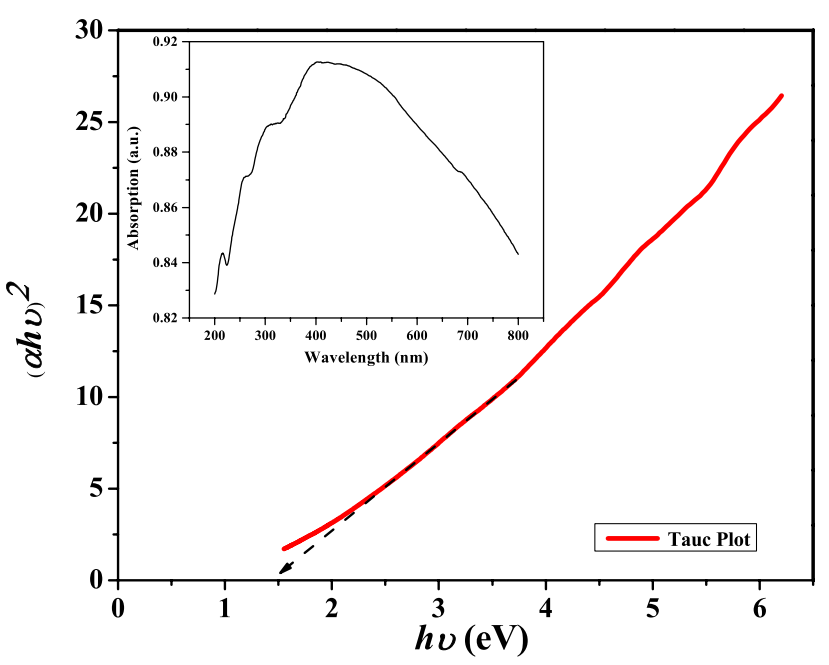

Fig. 4 Tauc plot for the determination of the optical band gap of the composite $0.7 \mathrm{BFO}-0.3 \mathrm{TMO}$ at room temperature. The black dashed arrow is to guide the eye, showing the extracted bad gap. The inset shows the absorption spectrum of the composite from which the Tauc plot has been estimated 

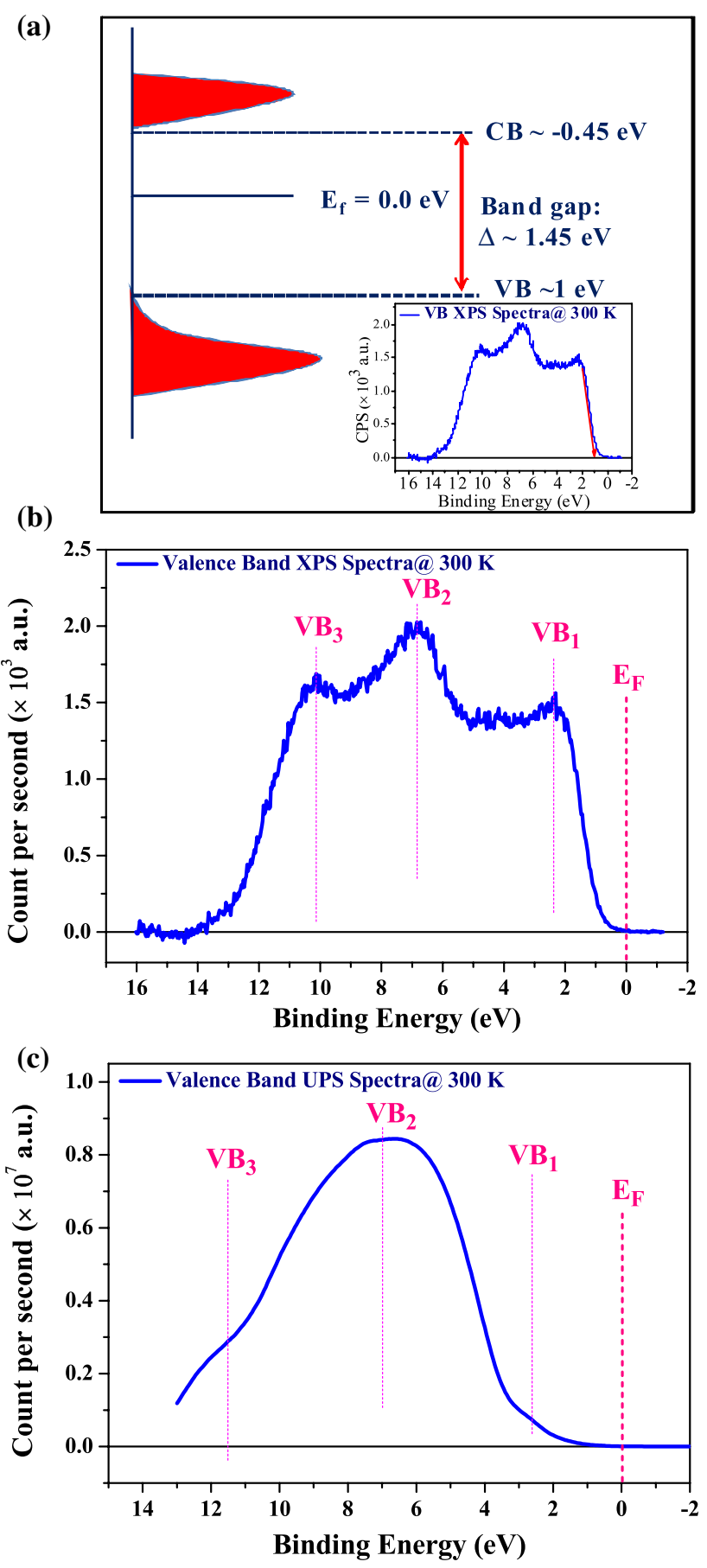

Fig. 5 a Schematic diagram of the band structure. Inset shows the XPAS valence spectra of the composite 0.7BFO-0.3TMO. The red line is to guide the eye showing the position of the edge of the valence band. $\mathbf{b}$ Valence band XPS spectrum of the composite at $300 \mathrm{~K}$. c UPS spectrum of the composite at room temperature

nature with high resistance (discussed later). Hence, our VB spectra corroborate well with our earlier results. The energy position of the valence band maximum has been estimated to be $\sim 1 \mathrm{eV}$ by making a linear extrapolation of the sharply rising feature immediately below the Fermi level (as shown in inset of Fig. 5a) $[45,51]$. Apart from this, knowing the band gap of the system $(\sim 1.45 \mathrm{eV})$ and the valence band maximum energy position $(\sim 1 \mathrm{eV})$, the position of the conduction band (CB) minimum can be readily determined to be $\sim-0.45 \mathrm{eV}$. Hence, a schematic diagram of its possible density of states (DOS) (band structure) is depicted in Fig. 5 a.

Furthermore, three main features of XPS VB spectra below the $E_{\mathrm{F}}$ can be observed at $\sim 2.5 \mathrm{eV}, 7 \mathrm{eV}$, and $10.5 \mathrm{eV}$, which are denoted as $\mathrm{VB}_{1}, \mathrm{VB}_{2}$, and $\mathrm{VB}_{3}$ respectively. The complete occupied $\mathrm{VB}$ spectra below $E_{\mathrm{F}}$ (ranging from $0 \mathrm{eV}$ to $\sim 14 \mathrm{eV}$ ) is mainly composed of hybridized states of Tb4f, extended Mn3d, Fe3d, and O2p orbitals. Since, for the present system, the $\mathrm{Mn}$ and Fe are in octahedral co-ordination with ligand oxygen ions, the crystal field effect causes the $\mathrm{Mn} / \mathrm{Fe} 3 d$ states to split into $e_{g}$ and $t_{2 g}$ levels. Moreover, for such kind of co-ordination, the $t_{2 g}$ states always lie below the $e_{\mathrm{g}}$ states. Therefore, the spectral weight (in the range of $0 \mathrm{eV}$ to $\sim 1 \mathrm{eV}$ ) immediately below $E_{\mathrm{F}}$ can be mainly attributed to the contributions from partially occupied Mn3d_ $e_{g}$ and Fe3d_e $e_{g}$ orbitals [46]. However, the first shoulder like feature $\mathrm{VB}_{1}$ positioned at $\sim 2.5 \mathrm{eV}$ has appeared mainly due to the hybridization of Tb4f, extended Mn3d_t $t_{2 g}$, and Fe3d_t $t_{2 g}$ with $\mathrm{O} 2 p$ orbitals. However, a significant contribution to this feature $\mathrm{VB}_{1}$ is expected to be from $\mathrm{Tb} 4 \mathrm{f}$ states as the other states $\left(\mathrm{Mn} 3 d_{-} t_{2 g}, \mathrm{Fe} 3 d_{-} t_{2 g}\right)$ are extended over a long range [52]. The most intense feature of the VB spectra at $\sim 7 \mathrm{eV}$ $\left(V_{2}\right)$ can be attributed mainly to the hybridization of the Mn3d_t $t_{2 g}$ and Fe3d_t $t_{2 g}$ orbitals with the $02 p$ states. On the other hand, the feature $\mathrm{VB}_{3}$ at the lowest energy $\sim 10.5 \mathrm{eV}$ emanates from the hybridized states of $\mathrm{O} 2 p-\mathrm{Mn} 3 d_{-} t_{2 g} /$ $\mathrm{Fe} 3 d_{-} t_{2 g}$ and other oxygen bonding states $\mathrm{O} 2 p-\mathrm{Mn} / \mathrm{Fe} 4 s p$ and $\mathrm{O} 2 p-\mathrm{Tb} 5 s d$, etc., with significant contribution being from $02 p$ states [52].

Moreover, to probe how the VB spectral features get modified with higher resolution and also to augment with our previous results of a electronic structure near Fermi level, we have carried out the ultraviolet photoemission spectroscopy (UPS) measurement on the same system (Fig. 5c). It is interesting to note here that the spectral features in UPS VB spectra immediately below the $E_{\mathrm{F}}$ are of relatively low intensity as compared to those observed in XPS VB spectra. This in turn predicts the fact that the region immediately below $E_{\mathrm{F}}$ has significant contribution from the $\mathrm{Mn} / \mathrm{Fe} 3 d$ states since the photo-ionization cross-section for the $\mathrm{Mn} / \mathrm{Fe} 3 d$ photoemission is considerably higher than that for the $\mathrm{O} 2 p$ photoemission in the XPS process. As a consequence, the low-energy UPS is less sensitive to the heavier atoms and highly sensitive to the lighter atoms such as oxygen. Hence, in the whole UPS spectra, the predominant contribution comes from the 
$\mathrm{O} 2 p$ states, while the contributions from the $\mathrm{Tb} 4 f, \mathrm{Mn} 3 d$, and Fe $3 d$ states get suppressed. Irrespective of the above facts, the UPS VB spectra agreed mostly with the XPS VB spectra. The absence of the electronic states near the Fermi level in the UPS VB spectra supports our previous results, thus suggesting its insulating nature. Moreover, a feature $\left(\mathrm{VB}_{1}\right)$ near $\sim 2.5 \mathrm{eV}$ (associated with the hybridized states of $\mathrm{O} 2 p-\mathrm{Mn} / \mathrm{Fe} 3 d$ and $\mathrm{O} 2 p-\mathrm{Tb} 4 f$ orbitals) can be visible in the UPS VB spectra which corroborate with the XPS $V B$ spectra. Similarly, the second feature $\left(V_{2}\right)$ which is the intense broad peak near $\sim 7 \mathrm{eV}$ matches well with the most intense peak of the XPS VB spectra. Another weak feature $\left(\mathrm{VB}_{3}\right)$ near $\sim 11.5 \mathrm{eV}$ can also be visible which is seemingly associated with the $\mathrm{O} 2 p-\mathrm{Mn} / \mathrm{Fe} 3 d$ hybridized states with significant contribution coming from $\mathrm{O} 2 p$ states. The position of the last feature differs by $\sim 1 \mathrm{eV}$ with that of the $\mathrm{VB}_{3}$ feature observed in XPS VB spectra. This can presumably be attributed to the possible charging effect due to the high resistivity of the system.

In our earlier study and in other works, it has been found that the $\mathrm{BiFeO}_{3}$-based materials show high dielectric constant and magnetodielectric coupling $[21,22,30]$. Hence, it is significant to study the transport property of the composite. Figure 6 shows the resistivity vs. temperature plot of the composite measured in the temperature range $10-300 \mathrm{~K}$. The resistivity of the sample increases continuously with decreasing temperature indicating semiconducting nature of the composite. This also indicates the involvement of activation process in the transport mechanism. Below $42 \mathrm{~K}$, the resistivity of the sample increases sharply. The inset (a) of Fig. 6 shows In $\rho$ versus $1000 / T$ plot signifying Arrhenius fitting which shows that our data poorly fit the Arrhenius equation. Hence, the

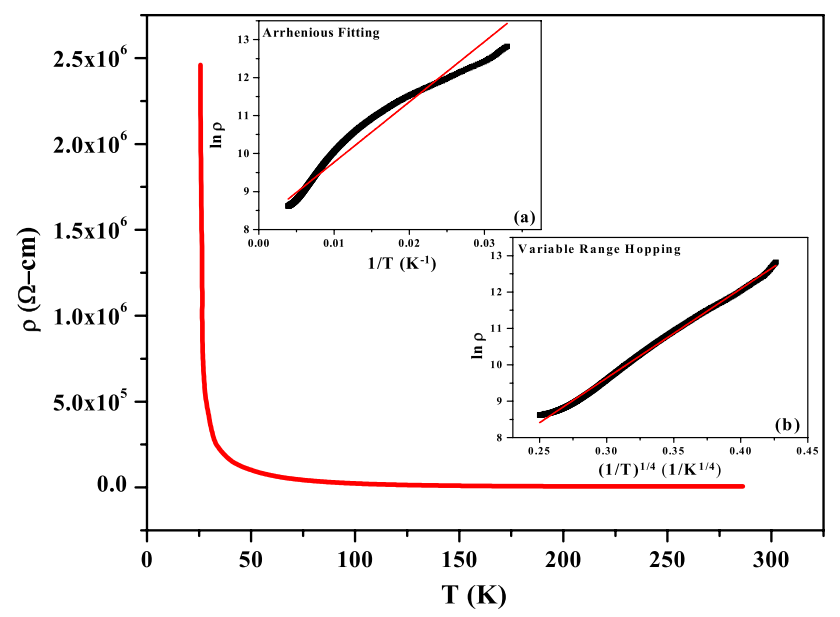

Fig. 6 Temperature-dependent resistivity of the composite 0.7BFO-0.3TMO. Inset a In $\rho$ versus $1 / T$ plot showing Arrhenius plot. Inset $\mathbf{b} \ln \rho$ versus $(1 / T)^{1 / 4}$ showing the plot for variable-range hopping
Mott's variable-range hopping (VRH) mechanism was considered to govern the transport process. The resistivity in case of VRH model can be written as: [53]

$\rho=\rho_{0} \exp \left[\left(\frac{T_{M}}{T}\right)^{\frac{1}{4}}\right]$,

where $T_{\mathrm{M}}$ is known as Mott's characteristic temperature and is expressed as:

$T_{\mathrm{M}}=21.2 \alpha^{3} / k_{\mathrm{B}} N\left(E_{\mathrm{F}}\right)$.

The most probable hopping distance $(R)$ and the hopping energy $(W)$ can be presented as:

$R=\left[8 \pi \alpha N\left(E_{\mathrm{F}}\right) k_{\mathrm{B}} T / 9\right]^{-\frac{1}{4}}$,

$W=3 / 4 \pi R^{3} N\left(E_{\mathrm{F}}\right)$,

where $1 / a$ is the localization length. The average ionic radii of the two transition metal ions $\left(\mathrm{Fe}^{3+}\right.$ and $\left.\mathrm{Mn}^{3+}\right)$ which are considered responsible for the conduction mechanism are taken as the localization length. Inset (b) of Fig. 6 shows the $\ln \rho$ versus $(1 / T)^{1 / 4}$ plot, which displays a good linear fitting thus supports the transport mechanism to be VRH. It is noteworthy to mention here that in disordered systems with random distribution of defects, VRH mechanism can generally be found to control the transport process [54]. In our sample, there are site defects due to the presence of mixed valence of $\mathrm{Mn}$ ions. The large strain produced at the interface of the two materials due to lattice mismatch would definitely create lattice defects in the composite system. Moreover, the high resistivity of the sample is consistent with band gap ascribed from the UV-visible spectrum and the band structure calculated from the VB XPS and UPS spectra.

To gain knowledge about the effect of band reconstruction and creation of different valence state of $\mathrm{Mn}$ ions on the magnetic property of the composite, we have measured the room-temperature magnetization $(M)$ versus magnetic field $(\mathrm{H})$ hysteresis loop of the composite and compared with that of pure BFO. Enhancing the magnetic property of $\mathrm{BiFeO}_{3}$ is one of the most significant challenges in multiferroic material study as it is the only multiferroic whose ordering temperatures (both magnetic and electric) are above the room temperature. Figure 7 presents the measured $\mathrm{M}-\mathrm{H}$ loops for the magnetic field $\pm 7 \mathrm{~T}$ from which it can be seen that the magnetization increases linearly with the applied field. This unsaturated behavior is consistent with the antiferromagnetic nature of $\mathrm{BiFeO}_{3}$ at room temperature. A closer look around the zero field reveals that at low fields there is deviation from 


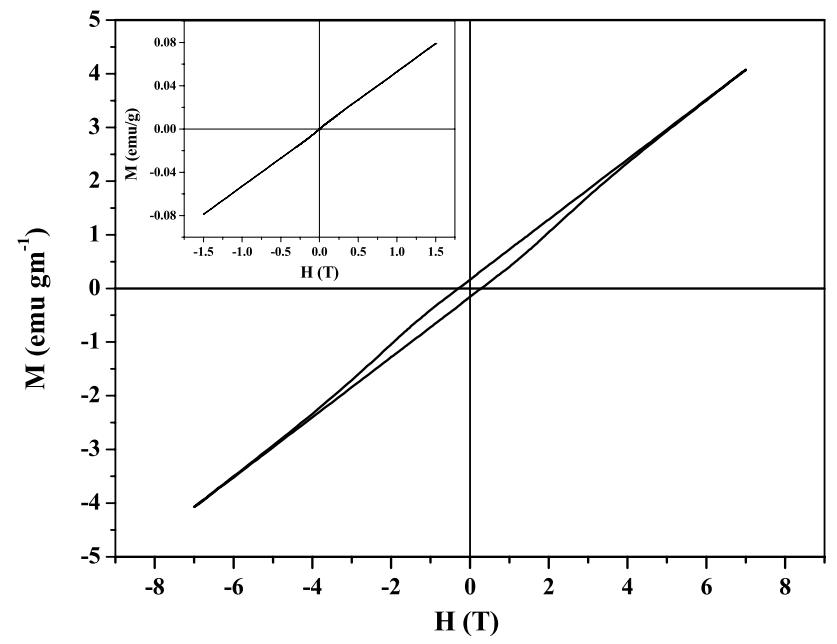

Fig. 7 Room-temperature $\mathrm{M}-\mathrm{H}$ loop of the composite 0.7BFO0.3TMO. Inset shows the room temperature $\mathrm{M}-\mathrm{H}$ loop of pure $\mathrm{BiFeO}_{3}$

the linearity and the loop is wide open. This along with the loop opening even at high fields signifies ferromagnetic contribution in the magnetic property of the sample. There are reports on the room-temperature weak ferromagnetism in the nanoparticles of $\mathrm{BiFeO}_{3}[8]$. Also $\mathrm{BiFeO}_{3}$ is known to possess canted antiferromagnetic ordering with a cycloid frequency $\sim 62 \mathrm{~nm}$ which is governed by the Dzyaloshinskii-Moriya interaction $[5,6,8]$. The weak ferromagnetism can also arise from the canted antiferromagnetic ordering. Inset in Fig. 7 shows the $\mathrm{M}-\mathrm{H}$ loop for the pure BFO sample. It can be seen that the magnetization of $\mathrm{BFO}$ also does not saturate showing its antiferromagnetic nature. Interestingly, it can be noticed that the magnetization value increases by an order of two. The coercivity of the material is found to increase from that of pure BFO. The increase and the low field weak ferromagnetic behavior can be understood to be due to the possible ferromagnetic superexchange interaction between different $\mathrm{Mn}$ ions $\left(\mathrm{Mn}^{2+}, \mathrm{Mn}^{3+}\right.$, and $\mathrm{Mn}^{4+}$ as seen from XPS spectra) at the interface. According to Goodenough-Kanamori rules the superexchange interactions between $\mathrm{Fe}^{3+}-\mathrm{O}-\mathrm{Mn}^{2+}$ / $\mathrm{Mn}^{4+}$ are also expected to be ferromagnetic $[26,55,56]$. It is difficult to estimate the contribution of different interactions which dominate the magnetic behavior. However, from the XPS study, it is observed that the relative fractions are found to be $22.85 \%, 50.46 \%$, and $26.68 \%$ for the $\mathrm{Mn}^{2+}, \mathrm{Mn}^{3+}$, and $\mathrm{Mn}^{4+}$ respectively. $\mathrm{Mn}^{3+}$ dominates the fractions as expected, whereas $\mathrm{Mn}^{4+}$ and $\mathrm{Mn}^{2+}$ have also significant fractions. From the analysis, it can be concluded that $\mathrm{Fe}^{3+}$ ions are present in the sample in the largest amount among the transition metal $\mathrm{Mn}^{3+}, \mathrm{Mn}^{4+}$, and $\mathrm{Mn}^{2+}$ in decreasing order. Clearly, the most dominating interactions would be $\mathrm{Fe}^{3+}-\mathrm{O}-\mathrm{Fe}^{3+}$. Among the ferromagnetic interactions, $\mathrm{Fe}^{3+}-\mathrm{O}-\mathrm{Mn}^{3+}$ would be the strongest, and similarly, we can say that the superexchange interaction of $\mathrm{Mn}^{2+}-\mathrm{O}-\mathrm{Mn}^{4+}$ would be the weakest. Thus, the composite shows higher coercivity in comparison with pure $\mathrm{BiFeO}_{3}$.

\section{Conclusion}

To summarize, we have prepared the $\mathrm{BiFeO}_{3}-\mathrm{TbMnO}_{3}$ composite (7:3) via conventional solid-state reaction and studied the interface through different characterizations. Morphological detail and the grain growth were studied from the SEM images. Chemical state and the composition of the material were studied from XPS and EDXA which revealed that oxygen vacancy is there which in turn creates mix valence state of $\mathrm{Mn}$ ions to maintain the charge neutrality. A remarkable decrease in band gap was observed from the UV-visible absorption spectra from that of $\mathrm{BiFeO}_{3}$. Based on the band gap $(\sim 1.45 \mathrm{eV})$ and the results from XPS valence band spectra and UPS spectra, the band structure of the material was drawn in which the conduction band edge was found $\sim 0.45 \mathrm{eV}$. In the valence band, three main features were observed at binding energy positions $\sim 2.5 \mathrm{eV}, 7 \mathrm{eV}$, and $10.5 \mathrm{eV}\left(\mathrm{VB}_{1}, \mathrm{VB}_{2}\right.$, $\mathrm{VB}_{3}$, and $\mathrm{VB}_{4}$ ) which were composed of hybridized states of Tb4f, extended Mn3d, Fe3d, and O2p orbitals. The most intense feature $\left(\mathrm{VB}_{2}\right)$ was attributed to the hybridization of the Mn3d_t $t_{2 g}$ and Fe3d_ $t_{2 g}$ orbitals with the O2p states, while the weak shoulder-like feature $\mathrm{VB}_{1}$ which close to the Fermi energy is attributed to the Tb4f states. Such band diagram and the reduction in the band graph are due to the reconstruction of the bands due to interfacial strain. Moreover, the transport property was found to be dominated by variable-range hopping mechanism, and the high resistivity of the material was also found to be consistent with the band diagram. Antiferromagnetic like non-saturating $\mathrm{M}-\mathrm{H}$ loop was observed with weak ferromagnetic features at low fields which was attributed to the superexchange interaction between different $\mathrm{Mn}$ ions.

Acknowledgements Authors would like to acknowledge the central instrument facility center of IIT (BHU) for SEM, EDXA, and magnetic measurements.

\section{Compliance with ethical standards}

Conflict of interest On behalf of all authors, the corresponding author states that there is no conflict of interest.

\section{References}

1. Eerenstein W, Mathur ND, Scott JF (2006) Multiferroic and magnetoelectric materials. Nature 442:759-764 
2. Fiebig MJ (2005) Revival of the magnetoelectric effect. J Phys D Appl Phys 38:R123-R152

3. VanAken BB, Rivera JP, Schmid H, Fiebig M (2007) Observation of ferrotoroidic domains. Nature 449:702-705

4. Xu G, Wen J, Stock C, Gehring PM (2008) Phase instability induced by polar nanoregions in a relaxor ferroelectric system. Nat Mater 7:562

5. Wang J, Neaton JB, Zheng $H$, Nagarajan V, Ogale SB, Liu B, Viehland D, Vaithyanathan V, Schlom DG, Wuttig M, Ramesh R (2003) Epitaxial $\mathrm{BiFeO}_{3}$ multiferroic thin film heterostructures. Science 299:1719-1722

6. Fischer P, Polomska M, Sosnowska I, Szymanksi M (1980) Temperature dependence of the crystal and magnetic structures of $\mathrm{BiFeO}_{3}$. J Phys C Condens Matter 13:1931-1940

7. Tabares-Munoz C, Rivera JP, Bezinges A, Monnier A, Schmid H (1985) Measurement of the quadratic magnetoelectric effect on single crystalline $\mathrm{BiFeO}_{3}$. Jpn J Appl Phys Part 1 24:1051-1053

8. ParkTJ, Papaefthymiou GC, Viescas AJ, Moodenbaugh AR, Wong SS (2007) Size-dependent magnetic properties of single-crystalline multiferroic $\mathrm{BiFeO}_{3}$ nanoparticles. Nano Lett 7:766-772

9. Kang YQ, Cao MS, Yuan J, Shi XL (2009) Microwave absorption properties of multiferroic $\mathrm{BiFeO}_{3}$ nanoparticles. Mater Lett 63:1344-1346

10. Yuana J, Houb ZL, Yanga HJ, Lia Y, Kanga YQ, Songa WL, Jina HB, Fanga XY, Cao MS (2013) High dielectric loss and microwave absorption behavior of multiferroic $\mathrm{BiFeO}_{3}$ ceramic. Ceram Int 39:7241-7246

11. Selbach SM, Einarsrud MA, Grande T (2009) On the thermodynamic stability of $\mathrm{BiFeO}_{3}$. Chem Mater 21:169-173

12. Rojac T, Kosec M, Budic B, Setter N, Damjanovic D (2010) Strong ferroelectric domain-wall pinning in $\mathrm{BiFeO}_{3}$ ceramics. J Appl Phys 108:074107

13. Rojac T, Kosec M, Damjanovic D (2011) Large electricfield induced strain in $\mathrm{BiFeO}_{3}$ ceramics. J Am Ceram Soc 94:4108-4111

14. Ramachandran B, Dixit A, Naik R, Lawes G, Rao MSR (2012) Weak ferromagnetic ordering in $\mathrm{Ca}$ doped polycrystalline $\mathrm{BiFeO}_{3}$. J Appl Phys 111:023910

15. Cheng ZX, Li AH, Wang XL, Dou SX, Ozawa K, Kimura H, Zhang SJ, Shrout TR (2008) Structure, ferroelectric properties, and magnetic properties of the La-doped bismuth ferrite. J Appl Phys 103:07E507

16. Yuan GL, Or SW, Liu JM, Liu ZG (2006) Structural transformation and ferroelectromagnetic behavior in single-phase $\mathrm{Bi}_{1-x} \mathrm{Nd}_{x} \mathrm{FeO}_{3}$ multiferroic ceramics. Appl Phys Lett 89:052905

17. Li Y, Zhang H, Li Q, Liu H, Dong X, Mao W, Dong C, Liu S, Li X, Wei $S$ (2012) Structural distortion and room-temperature ferromagnetization of Co-doped and (Eu, Co)-co doped $\mathrm{BiFeO}_{3}$ nanoparticles. Mater Lett 87:117-120

18. Xu J, Xie D, Yin C, Feng T, Zhang X, Zhao H, Li G, Ren TL, Guan Y, Gao X, Pan W (2014) Thick film patterning by lift-off process using double-coated single photoresists, Mg-doped $\mathrm{Bi}_{0.8} \mathrm{Ca}_{0.2} \mathrm{FeO}_{3}$ with enhanced ferromagnetic properties. Mater Lett 122:139-142

19. Li Y, Cao W, Yuan J, Wang D, Cao M (2015) Nd doping of bismuth ferrite to tune electromagnetic properties and increase microwave absorption by magnetic-dielectric synergy. J Mater Chem C 3:9276

20. Li ZJ, Hou ZL, Song WL, Liu XD, Cao WQ, Shao XH, Cao MS (2016) Unusual continuous dual absorption peaks in Ca-doped $\mathrm{BiFeO}_{3}$ nanostructures for broadened microwave absorption. Nanoscale 8:10415

21. Li Y, Fang X, Cao M (2016) Thermal frequency shift and tunable microwave absorption in $\mathrm{BiFeO}_{3}$ family. Sci Rep 6:24837

22. Li Y, Cao M, Wanga D, Yuan J (2015) High-efficiency and dynamic stable electromagnetic wave attenuation for $\mathrm{La}$ doped bismuth ferrite at elevated temperature and gigahertz frequency. RSC Adv 5:77184

23. Singh A, Patel JP, Pandeya D (2009) High temperature ferroic phase transitions and evidence of paraelectric cubic phase in the multiferroic $0.8 \mathrm{BiFeO}_{3}-0.2 \mathrm{BaTiO}_{3}$. Appl Phys Lett 95:142909

24. Basu A, Jana R, Ranjan R, Mukherjee GD (2016) Pressure effects on model ferroelectric $\mathrm{BiFeO}_{3}-\mathrm{PbTiO}_{3}$ : multiple phase transitions. Phys Rev B 93:214114

25. Choi EM, Kleibeuker JE, Fix T, Xiong J, Kinane CJ, Arena D, Langridge $\mathrm{S}$, Chen A, Bi Z, Lee JH, Wang $\mathrm{H}$, Jia Q, Blamire MG, MacManus-Driscoll JL (2016) Interface-coupled $\mathrm{BiFeO}_{3} / \mathrm{BiMnO}_{3}$ superlattices with magnetic transition temperature up to $410 \mathrm{~K}$. Adv Mater Interfaces 3:1500597

26. Yu P, Lee JS, Okamoto $S$, Rossell MD, Huijben $M$, Yang $C H, H e ~ Q$, Zhang JX, Yang SY, Lee MJ, Ramasse QM, Erni R, Chu YH, Arena DA, Kao CC, Martin LW, Ramesh R (2010) Interface ferromagnetism and orbital reconstruction in $\mathrm{BiFeO}_{3}-\mathrm{La}_{0.7} \mathrm{Sr}_{0.3} \mathrm{MnO}_{3}$ heterostructures. Phys Rev Lett 105:027201

27. Kimura T, Goto T, Shintani H, Ishizaka K, Arima T, Tokura Y (2003) Magnetic control of ferroelectric polarization. Nature 426:55-58

28. Prokhnenko O, Aliouane N, Feyerherm R, Dudzik E, Wolter AUB, Maljuk A, Kiefer K, Argyriou DN (2010) Ga substitution as an effective variation of $\mathrm{Mn}$-Tb coupling in multiferroic $\mathrm{TbMnO}_{3}$. Phys Rev B 81:024419

29. Kubel F, Schmid H (1990) Structure of a ferroelectric and ferroelastic monodomain crystal of the perovskite $\mathrm{BiFeO}_{3}$. Acta Crystallogr Sect B 46:698

30. Gupta PK, Ghosh S, Kumari S, Pal A, Roy S, Singh R, Singh P, Singh RK, Ghosh AK, Chatterjee S (2019) Spin phonon coupling and magneto-dielectric coupling in $\mathrm{BiFeO}_{3}-\mathrm{TbMnO}_{3}$ composite. Mater Res Express 6:086114

31. Kumar A, Shahi P, Kumar S, Shukla KK, Singh RK, Ghosh AK, Nigam AK, Chatterjee S (2013) Raman effect and magnetic properties of doped $\mathrm{TbMnO}_{3}$. J Phys D Appl Phys 46:125001

32. Bammannavar BK, Naik LR, Chougule BK (2008) Studies on dielectric and magnetic properties of $(x) \mathrm{Ni}_{02} \mathrm{Co}_{08} \mathrm{Fe}_{2} \mathrm{O}_{4}+(1-\mathrm{x})$ barium lead zirconate titanate magnetoelectric composites. $J$ Appl Phys 104:064123

33. Mukherjee A, Hossain SM, Pal M, Basu S (2012) Effect of Y-doping on optical properties of multiferroics $\mathrm{BiFeO}_{3}$ nanoparticles. Appl Nanosci 2:305-310

34. Wu J, Wang J (2010) Ferroelectric and Impedance Behavior of La- and Ti-Codoped BiFeO3 Thin Films. J Am Ceram Soc 93:2795-2803

35. Adhlakha N, Yadav KL, Singh R (2015) $\mathrm{BiFeO}_{3}-\mathrm{CoFe}_{2} \mathrm{O}_{4}-\mathrm{PbTiO}_{3}$ composites: structural, multiferroic, and optical characteristics. J Mater Sci 50:2073-2084

36. Mishra AK, Ramaprabhu S (2011) Nano magnetite decorated multiwalled carbon nanotubes: a robust nanomaterial for enhanced carbon dioxide adsorption. Energy Environ Sci 4:889-895

37. Chauhan S, Kumar M, Chhoker S, Katyal SC, Singh M (2016) Substitution driven structural and magnetic transformation in Cadoped $\mathrm{BiFeO}_{3}$ nanoparticles. RSC Adv 6:43080-43090

38. Fujii T, Groot FMF, Sawatzky GA, Voogt FC, Hibma T, Okada K (1999) In situ XPS analysis of various iron oxide films grown by $\mathrm{NO}_{2}$-assisted molecular-beam epitaxy. Phys Rev B 59:3195

39. Ghosh S, Srivastava N, Srivastava PC (2014) Injecting electrode controlled electronic transport across $\mathrm{Fe}_{3} \mathrm{O}_{4}$ film-Si interfacial structure. J Alloys Compd 612:418-424

40. Di L, Yang $H$, Xian T, Chen $X$ (2017) Enhanced photocatalytic activity of $\mathrm{NaBH}_{4}$ reduced $\mathrm{BiFeO}_{3}$ nanoparticles for rhodamine $B$ decolorization. Materials 10:1118

41. Kang JS, Lee HJ, Kim G, Kim DH, Dabrowski B, Kolesnik S, Lee H, Kim JY, Min BI (2008) Electronic structure of the cubic perovskite 
$\mathrm{SrMn}_{1-\mathrm{x}} \mathrm{Fe}_{\mathrm{x}} \mathrm{O}_{3}$ investigated by X-ray spectroscopies. Phys Rev B 78:054434

42. Kim MS, Yang JB, Cai Q, Zhou XD, James WJ, Yelon WB, Parris PE, Buddhikot D, Malik SK (2005) Structure, magnetic, and transport properties of Ti-substituted $\mathrm{La}_{0.7} \mathrm{Sr}_{0.3} \mathrm{MnO}_{3}$. Rev B 71:014433

43. Bharathkumar S, Sakar M, Vinod KR, Balakumar S (2015) Versatility of electrospinning in the fabrication of fibrous mat and mesh nanostructures of bismuth ferrite $\left(\mathrm{BiFeO}_{3}\right)$ and their magnetic and photocatalytic activities. Phys Chem Chem Phys 17:17745

44. Patel SKS, Lee J-H, Kim M-K, Bhoi B, Kim S-K (2018) Single-crystalline $\mathrm{Gd}$-doped $\mathrm{BiFeO}_{3}$ nanowires: $\mathrm{R3c}$-to-Pn21a phase transition and enhancement in high-coercivity ferromagnetismJ. Mater Chem C 6:526-534

45. Nistor C, Krull C, Mugarza A, Stepanow S, Stamm C, Soares M, Klyatskaya S, Ruben M, Gambardella P (2015) Exchange bias of $\mathrm{TbPc}_{2}$ molecular magnets on antiferromagnetic FeMn and ferromagnetic Fe films. Phys Rev B 92:184402

46. Calderón MJ, Liang S, Yu R, Salafranca J, Dong S, Yunoki S, Brey L, Moreo A, Dagotto E (2011) Magnetoelectric coupling at the interface of $\mathrm{BiFeO}_{3} / \mathrm{La}_{0.7} \mathrm{Sr}_{0.3} \mathrm{MnO}_{3}$ multilayers. Phys. Rev. B 84:024422

47. Tauc J, Grigorvici R, Yanca Y (1966) Optical properties and electronic structure of amorphous germanium. Phys Status Solidi 15:627-637

48. Ihlefeld JF, Podraza NJ, Liu ZK, Rai RC, Xu X, Heeg T, Chen YB, Li J, Collins RW, Musfeldt JL, Pan XQ, Schubert J, Ramesh R, Schlom DG (2008) Optical band gap of $\mathrm{BiFeO}_{3}$ grown by molecularbeam epitaxy. Appl Phys Lett 92:142908

49. Hengky C, Moya X, Mathur ND, Dunn S (2012) Evidence of high rate visible light photochemical decolourisation of Rhodamine
$\mathrm{B}$ with $\mathrm{BiFeO}_{3}$ nanoparticles associated with $\mathrm{BiFeO}_{3}$ photocorrosion. RSC Adv 2:11843

50. Zhang X, Liu H, Zheng B, Lin Y, Liu D, Nan CW (2013) Photocatalytic and magnetic behaviors observed in $\mathrm{BiFeO}_{3}$ nanofibers by electrospinning. J Nanomater 2013:917948

51. Kang Q, Cao J, Zhang Y, Liu L, Xu H, Ye J (2013) Reduced $\mathrm{TiO}_{2}$ nanotube arrays for photoelectrochemical water splitting. $J$ Mater Chem A 1:5766-5774

52. Dwivedi GD, Joshi AG, Kumar S, Chou H, Yang KS, Jhong DJ, Chan WL, Ghosh AK, Chatterjee S (2016) Electronic structure study of wide band gap magnetic semiconductor $\left(\mathrm{La}_{0.6} \mathrm{Pr}_{0.4}\right)_{0.65} \mathrm{Ca}_{0.35} \mathrm{MnO}_{3}$ nanocrystals in paramagnetic and ferromagnetic phases. Appl Phys Lett 108:172402

53. Shklovskii BI, Efros AL (1984) Electronic properties of doped semiconductors. Springer, Berlin

54. Wang H, Li G, Zhao M, Li L (2012) Spin state transition and giant dielectric constant in $\mathrm{Pr}_{0.98} 7 \mathrm{Na}_{0.013} \mathrm{CoO}_{3}$. Appl. Phys. Lett. 100:152109

55. Goodenough JB (1955) Theory of the role of covalence in the perovskite-type manganites $[\mathrm{La}, \mathrm{M}(\mathrm{II})] \mathrm{MnO}_{3}$. Phys Rev 100:564-573

56. Kanamori J (1959) Superexchange interaction and symmetry properties of electron orbitals. J Phys Chem Solids 10:87-98

Publisher's Note Springer Nature remains neutral with regard to jurisdictional claims in published maps and institutional affiliations. 